Please do not cite this preprint. A final, modified version of this article will be published as a chapter in the following edited volume:

García \& Ibáñez (to appear), Routledge Handbook of Neurosemiotics.

\title{
Experimental Semiotics: past, present, and future
}

Jonas Nölle ${ }^{1}{ }^{1} \&$ Bruno Galantucci (iD) 2

\begin{abstract}
Experimental Semiotics (ES) investigates how novel semiotic systems emerge by observing people ground communication in lab games, thus exposing the fundamental principles of human semiosis and the pressures through which they originate. We review the past of ES and describe its key findings. We then discuss challenges and future directions for ES, including how a tighter integration with neuroscience can enrich our understanding of the social processes exposed by ES, and how ES can in turn help neuroscience investigate human semiosis in more realistic settings. ES provides neurosemiotics with new tools to integrate the social and biological components of communication.
\end{abstract}

Keywords: experimental semiotics, language evolution, interaction, symbol grounding, design features

${ }^{1}$ School of Psychology \& Neuroscience, University of Glasgow

62 Hillhead St, Glasgow G12 8QB, Scotland, UK

ORCID: https://orcid.org/0000-0003-3546-2689

Email: jonas.noelle@glasgow.ac.uk

${ }^{2}$ Department of Psychology, Yeshiva University

500 West 185th street, New York, NY, 10033, USA

ORCID: https://orcid.org/0000-0002-3642-1371

Email: bruno.galantucci@yu.edu 


\section{Introduction}

We humans are distinct from other animals in that our life is permeated by constant creation and manipulation of semiotic systems such as languages, writing systems, or the pictograms we exchange through our phones (Cassirer, 1953; Deacon, 1997). How do such semiotic systems come about? Over the last two decades, research performed in 'Experimental Semiotics' (ES) has addressed this question empirically by studying in the lab how people develop novel semiotic systems.

In this article, we first situate ES within its historical context. We then describe a sample of the key scientific contributions ES has offered since its inception. These contributions have a broad scope, ranging from insights into the fundamentals of human semiosis to findings concerning its cognitive and social underpinnings. In other words, despite its recent origins, ES has already demonstrated a great potential as an engine of discovery in the cognitive, language, and social sciences. We conclude the article by discussing current challenges that ES faces, and how these can be approached in the future with novel technology and a tighter integration with neuroscientific methods, to the mutual benefit of both disciplines, in the best spirit of the neurosemiotic framework.

\section{Historical context of Experimental Semiotics}

Experimental Semiotics has both ancient and modern precursors. The question of how humans can create a novel communication system has sparked enquiries since at least $\sim 600 \mathrm{BC}$, when Pharao Psamtik I conducted an isolation experiment with two human infants to investigate whether language is innate. Such unethical experiments (usually resulting in severe damages for the infants) were repeated several times in history and can to some extent be considered precursors to ES as well as precursors to isolation studies into the innateness of bird song (e.g., Thorpe, 1958; Fehér et al., 2009). More modern precursors to laboratory experiments on communication can be traced back to psychologists such as Bartlett (1932) or Bavelas $(1950,1952)$, who studied in the lab how information transmission leads to the creation of 'stereotypical' memory patterns or the emergence of leadership. Although these scholars were interested in different questions, their paradigms resemble ES insofar they involve senders, receivers, and pressures concerning the processes through which people exchange information. Similarly, there have been early attempts at using artificial miniature languages to study language change (e.g., Esper, 1925; Wolfle, 1933) that in many ways resemble some of modern ES paradigms. Finally, recent precursors include strands of related research (Galantucci, 2017), such as Experimental Pragmatics (Noveck \& Sperber, 2004). In experimental pragmatic studies, participants use pre-existing forms of communication - i.e., their native language - to solve communicative problems. Such studies usually address how interlocutors design utterances to express their intention in a given context. Phenomena of interest are usually pragmatic aspects of communication such as relevance, degree of specificity, and how to efficiently structure utterances for successful communication (Grice, 1975; Gibson et al., 2019; Rubio-Fernandez \& JaraEttinger, 2020), often in the context of contemporary theoretical frameworks such as the $R a$ tional Speech-act Model (Frank \& Goodman, 2012; Goodman \& Frank, 2016). To study actual communication in the lab, these studies typically rely on communication tasks, i.e. tasks in which participants can succeed only if they communicate accurately about the entities of a specific experimental context (Krauss \& Weinheimer, 1964; Clark \& Wilkes-Gibbs, 1986; Horton \& Keysar, 1996). For instance, Garrod and colleagues used the Maze Game - where dyads have to communicate locations of gate-opening switches in two-dimensional mazes - to show that such problem-solving leads to routinisation of communicative conventions (Garrod 
\& Anderson, 1987). In a follow-up study, they found that these conventions would diffuse in a community of interacting partners (Garrod \& Doherty, 1994). The cooperative Maze Game has since been used in a number of studies addressing interactional phenomena such as alignment (Garrod \& Pickering, 2009), repair (Healey, 2008) or how linguistic conventions are shaped by environmental factors such as different maze configurations (Nölle, Fusaroli, et al., 2020).

The main difference between ES and experimental pragmatic studies is that ES focuses on the emergence of de novo communication systems in the lab. In other words, rather than allowing participants to communicate through their shared language, ES forces them to establish a novel semiotic system to solve the task at hand. For instance, in Galantucci's (2005) experiment, participants had to communicate locations in a similar vein to the Maze Game but could only communicate by drawing on a device that systematically distorted the drawings. In this respect, ES also resembles earlier attempts to simulate the emergence of linguistic structure or communication systems using formal mathematical models or populations of agents. Such models have been used to simulate cultural transmission of novel communicative signals as well as situations where such signals have to be established in interaction to achieve successful communication (e.g. Kirby \& Hurford, 2002; Brighton et al., 2005; Griffiths \& Kalish, 2007; Steels, 2011). One of the main achievements of ES studies has been to demonstrate that such research can also be conducted with humans to investigate how their own natural constraints shape the fundamental structures of communication (see also Box 1). While rigorously controlled, these experiments offer higher levels of ecological validity than computational models because they do not rely on ad-hoc assumptions concerning human nature (Galantucci \& Roberts, 2012) ${ }^{1}$.

Over the two decades that have elapsed since its inception, ES has offered a number of scientific contributions, from insights into the fundamentals of human semiosis, to discoveries concerning its cognitive and social underpinnings. In what follows, we will present and discuss a sample of these contributions. This sample is not meant as an exhaustive review. ES is a methodology that has been thriving after it was spontaneously developed in a few different scientific communities. Reviewing the full body of research generated thorough this methodology is beyond the scope of this article. Instead, we opted for presenting a sample of contributions which, in our opinion, best highlight the potential of ES as an engine of scientific discovery.

\section{The fundamentals of human semiosis}

Some of the early ES studies focused on fundamental aspects of human semiosis such as (a) how humans ground symbols and (b) how these symbols organise themselves in highly functional semiotic systems such as signed and spoken languages.

\subsection{Symbol grounding}

The origins of symbolic language have sometimes be referred to as the 'hardest problem in science' (Christiansen \& Kirby, 2003) that requires interdisciplinary efforts and a wide variety of empirical methods to be addressed (Fitch, 2017; Nölle, Hartmann, et al., 2020). Besides the biological, cognitive, and sociocultural prerequisites for human language (see, e.g., Fitch, 2010; Tomasello, 2008, 2014; Kirby, 2017), one of the central question concerns the 'symbol grounding problem' (Harnad, 1990): How does a population of agents that does not have a symbolic communication system ground such a system with non-symbolic means?

\footnotetext{
${ }^{1}$ Computational models, in turn, allow executing experiments that would be unethical or unpractical to perform with humans.
} 
Early ES studies by Galantucci (2005) and Garrod et al. (2007) provide two significant contributions to this question's answer. Firstly, they show that, in the context of joint actions with clear goals, humans can readily solve the symbol grounding problem. The second is that the problem itself provides a fertile experimental framework to study human semiotic behaviour under controlled laboratory conditions. In particular, these studies showed that, when participants who could not use their native language were asked to solve communication problems in a graphical communication medium, they would usually try to bootstrap signs based on motivated icons or indexical signals. Typically, these motivated forms were directly related to properties of (a) the task participants were performing or (b) the environment in which they were immersed. During the study, these initial forms would then gradually evolve into symbols. For instance, in Garrod et al.'s (2007) 'Pictionary' drawing task, initially participants tried to communicate referents by using very descriptive drawings such as a smoking cowboy to express 'Clint Eastwood'. These drawings tended to gradually evolve into simplified symbols (e.g., a circle and arrow indicating "East"), that were more efficient to produce but less transparent to outsiders (see also Box 1, Fig. 2). This process of 'symbolification' illustrates how ES studies offer an opportunity to observe the emergence of fundamental hallmarks of human symbolic systems such as arbitrariness and conventionality (de Saussure, 1916).

\subsection{Design features}

On first glance, one could question whether the highly abstract and simplified communication games in ES studies, which usually feature a single well-defined communicative objective and very restricted degrees of behavioural freedom, can provide a fertile terrain to grow something akin to a full-blown language in the lab. After all, the communication systems that emerge in such settings usually consist of very small inventories of signs that are just about sufficient to solve the task at hand (see Galantucci, Garrod, et al., 2012 for detailed examples). However, on the contrary, this restrictedness is one of the main strengths of ES because its experimental settings can be tailored to zoom in on a specific property of communication and isolate the variables the influence its emergence. In this way, ES studies have been able to investigate most of the 'design features' proposed by Hockett (1960) to capture the essence of human language as a semiotic system distinct from animal communication.

These include duality of patterning, i.e., the fact that languages rely on both combinatorial and compositional structure. Combinatoriality is the ability to combine discrete, meaningless forms into an indefinite number of meaningful combinations. In this way, spoken or signed languages use small inventories for making up words from combinations of phonemes or discrete features of manual gestures. Galantucci, Kroos and Rhodes (2010) showed that one pressure leading to the emergence of combinatoriality is another design feature, namely rapidity of fading, i.e. the fact that signed or spoken languages are evanescent (in contrast to, say, writing). They observed that participants dealing with more rapidly fading signals were more likely to reuse and combine simple elements to achieve communicative success. Further studies (Roberts et al., 2015; Little et al., 2017) have shown that arbitrariness likewise fosters combinatoriality because, when the meaning space (what is communicated) does not easily map onto the form space (how it is communicated), the pressure for efficient message transmission prevails over other communicative pressures. Since combinatorial signs greatly enhance efficient message transmission, people tend to prefer them over other sign types.

In addition to combinatoriality, human communication systems are also compositional and systematic (Dingemanse et al., 2015). They allow systematically recombining a finite set of existing meanings to express an open-ended set of possible meanings in the form of new words and sentences. Early computer simulations suggested that compositional structures could be the outcome of pressures from learning and communication acting upon communication systems as they are used and transmitted over generations (rather than derived from innate biology; 
see Brighton, 2002; Christiansen \& Chater, 2008). However, it was ES that allowed researchers to demonstrate for the first time that this emergence of structure could be observed in humans in the lab. In various studies, participants were given unstructured input, e.g., objects with random labels in an artificial language, or were asked to communicate a set of meanings with recurring dimensions (such as shape, colour, texture, etc.) in a novel communication medium (Kirby et al., 2014). A recurring finding has been that, over time, emerging communication systems become increasingly compositional in response to various pressures. For example, studies by Kirby and colleagues $(2008,2015)$ showed that initially holistic signals, where a random character string like tupim encodes a meaning like 'red triangle', could evolve into compositional labels in which, e.g., the first syllable encodes the shape, and the second syllable encodes the colour. This compositionality allows to express many possible shape-colour combinations with a finite set of meanings, similarly to how the English utterances green triangle, and blue triangle re-use a shared element. Systematic structure of this kind has been observed to emerge in different settings in studies by Theisen et al. (2010) and Motamedi et al. (2019) who had their participants communicate concepts belonging to specific semantic categories (e.g., writing, school, teacher, cooking, chef, restaurant) either though drawing or through gestures. In these experiments, certain elements of the drawings or single gestures people used to communicate would become re-used, so that for instance a 'cooking' gesture could be combined with a systematic gesture marking 'building' or 'person' to express restaurant or chef. A related gesture study by Nölle et al. (2018) showed that this tendency to evolve markers for systematic categories was amplified in an open-ended environment, where new meanings were constantly introduced (see also Selten \& Warglien, 2007; Carr et al., 2017). The study further found that another design feature, displacement, had an impact on the degree of systematicity: Dyads communicating about absent entities were more likely to introduce systematic gestures, presumably because categorising referents made it easier to reason about the task and set of target referents in working memory.

Together, these findings suggest that ES does not only allow studying design features of communication systems such as combinatoriality, rapidity of fading, systematicity, or displacement, but also to gain insights into their interrelations. Furthermore, ES has proven to be an excellent tool to study other aspects of human semiosis. For instance, some studies have tested how communication systems developed in the lab adapt to the communicative situation in which they are used, and found that different contexts determine the extent to which signals become holistic, underspecified, or systematic (Silvey et al., 2015; Winters et al., 2015; Nölle et al., 2018). 


\section{The cognitive and social underpinnings of human semiosis}

Thanks to ES methods, researchers have performed a host of studies aimed at uncovering, through experimental manipulations, the cognitive and social underpinnings of human semiosis. We describe a sample of these studies, focusing on sets of them that have emerged as more or less coherent lines of research in the literature.

\subsection{Cognitive underpinnings: Impact of modality}

Graphical media like drawing or gesture provide a strong affordance for iconicity, since they allow representing concepts via forms that resemble their referents. Some authors have therefore argued that gesture is better suited for bootstrapping communication systems (Fay et al., $2013,2014)$. However, how effectively a meaning can be represented iconically in a specific medium also depends on its semantics. While manual gestures can easily depict actions or objects, e.g., emotions can be easily expressed by facial expressions or vocalisations (Perlman et al., 2015; Perlman \& Lupyan, 2018). In the language sciences, it is becoming increasingly accepted that human communication is multi-modal and relies on large variety of integrated communication channels such as speech, gesture and facial expressions (Perniss, 2018; Holler \& Levinson, 2019) and ES can help tease apart the contributions of these channels and how they became integrated in the first place (see, e.g., Zlatev et al., 2017). Paradigms affording iconicity further lend themselves to the study of more specific aspects of language in detail, since the medium allows participants to quickly and reliably ground communication systems in the lab. Thus, silent gesture experiments have recently become a popular tool for studying the origins of specific grammatical structures like, e.g., cross-linguistic word order preferences that seem to reflect cognitive processes that can be observed in the lab (Goldin-Meadow et al., 2008; Schouwstra \& de Swart, 2014; Ortega \& Özyürek, 2020; Culbertson et al., 2020). Additionally, the manual modality is of special interest, since recently emerged natural sign-languages show similar evolutionary stages as gesture systems that emerge in the lab, such as high degrees of iconicity in early generations of users and the gradual development of discrete combinatorial elements that become organised in a system (Senghas, 2004; Fusellier-Souza, 2006; Motamedi et al., 2019). Here, lab experiments offer not just a parallel to naturalistic data but can help shed light on the underlying mechanisms that lead to the emergence of structure. This includes, e.g., the lab observations that prevalent iconicity can impede combinatoriality (Roberts \& Galantucci, 2012; Roberts et al., 2015; Little et al., 2017), which are reflected in the real-world case of Al Sayyid Bedouin Sign Language that developed in an isolated village community (Sandler et al., 2011), or the finding that spatial modulation to differentiate event arguments across discourse requires several generations of signers to develop, which has been attested both in the lab (Motamedi et al., 2018) and in Nicaraguan Sign Language (Kocab et al., 2015).

Other communicative modalities frequently used in ES include written miniature languages comprising small lexicons of artificial words (e.g., Kirby et al., 2008, 2015; Winters et al., 2015; Tinits et al., 2017; Smith et al., 2017), sequences of symbols such as letters or pictograms (e.g., Selten \& Warglien, 2007; Ruiter et al., 2010; Winters \& Morin, 2019) or different types of trackpads, touchscreens and motion control (e.g., Galantucci, 2005; Eryilmaz \& Little, 2017; Morin et al., 2018). To evoke complex structure, like duality of patterning, experiments need to include both a meaning space that affords compositional reference (e.g., recurring meaning dimensions such as colour, shape etc), and a continuous signalling space that affords creating both holistic and combinatoric messages. This has for instance be achieved with a slide whistle (Verhoef, 2012; Verhoef et al., 2014) or the Leap Motion device (Eryilmaz \& Little, 2017), which allow for discrete patterns to emerge from an initially continuous and unstructured signal. 


\subsection{Social underpinnings: Horizontal and vertical transmission}

Over the years, ES researchers have developed a wide range of experimental paradigms to address different questions in the field (see also Tamariz \& Kirby, 2016, p. 39). Early ES studies mainly relied on one of two basic designs: Firstly, dyadic interaction, where two participants solve a coordination problem (e.g., Galantucci, 2005; Scott-Phillips et al., 2009) or perform a referential communication task (Garrod et al., 2007). As we described above, this kind of studies have provided insights on how humans could bootstrap symbolic communication in the first place. Secondly, so called 'iterated learning' studies, where an initial 'generation' $n$ of participants is trained on an artificial language and has to reproduce it. Next, their output becomes the input for the subsequent generation $n+1$ and so forth, hence allowing to observe how the system evolves over time in reaction to learning biases present in the learners (see Kirby et al., 2014 for a review).

These different approaches led to the realisation that different types of interaction and population dynamics affect communication systems in different ways (Fay et al., 2010; Smith et al., 2013), which resulted in refinement of these design and further paradigms (Figure 1).

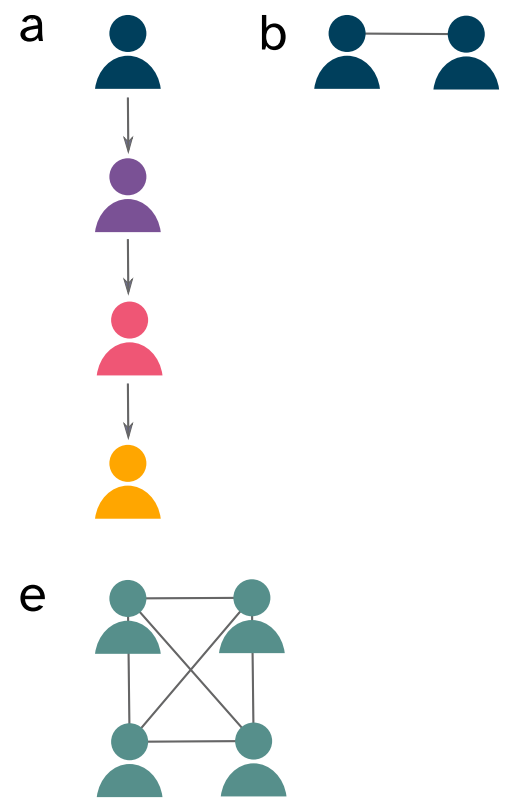

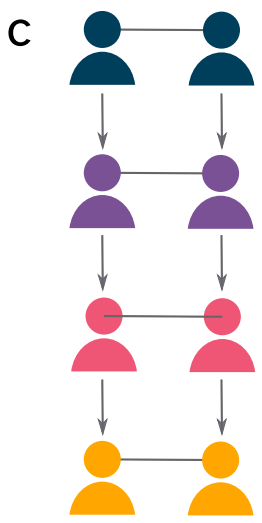

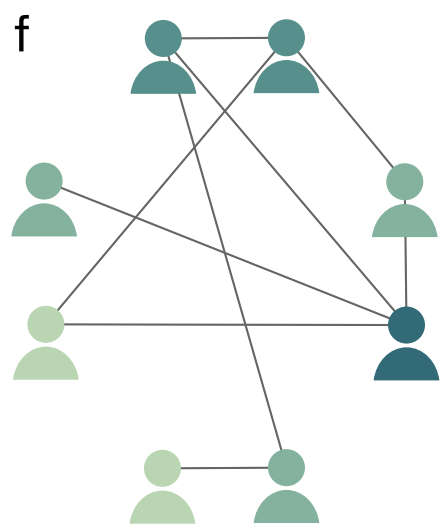

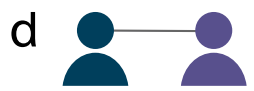
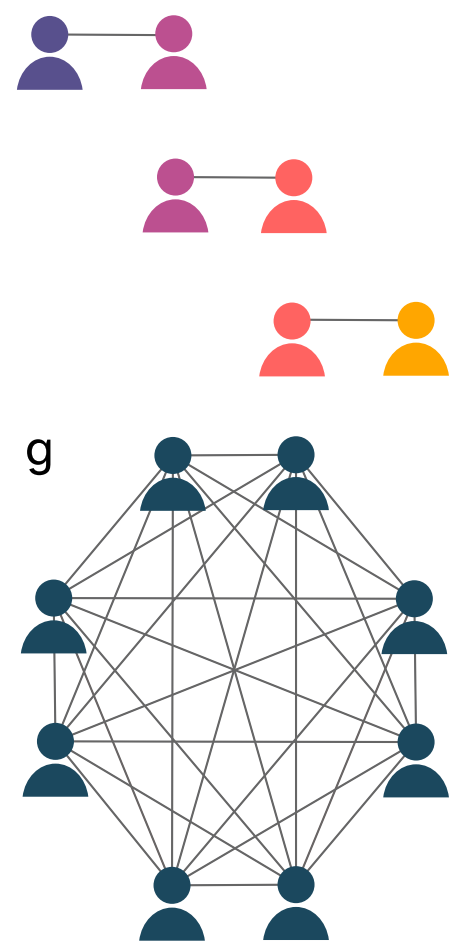

Figure 1: Common experimental designs used in ES studies. Arrows indicate vertical transmission; solid lines indicate horizontal interaction. a) Transmission only: An iterated learning chain modelling cultural transmission of a communication system over four generations). b) Dyadic interaction only: pairs of participants solve a communication game in each experimental session). c) Transmission and interaction: Dyads interact within an iterated chain, where the output of a dyad becomes the input for the next generation. d) Replacement method: Dyads interact, and after some time one dyad member is replaced with a new participant, simulating population turnover (in this case, five participants in total). e-f) Microsocieties: Participants interact with several members of an artificial 'community' to simulate the spread of variants in a population. Different population sizes (e.g., e vs f) and social network topologies (e.g., fully connected networks in $\mathbf{e}$ and $\mathbf{g}$ vs a sparse network in $\mathbf{f}$ ) allow studying how population dynamics affect the evolution of the communication systems that emerge in these settings. 
Generally, interaction puts communicative pressures on signalling systems. Signals need to be expressive and informative, so that interlocutors can readily interpret what their partner is signalling to solve the task at hand, while also being simple and efficient so as to reduce production costs and facilitate communication. Pairs in dyadic-only studies therefore often settle on very efficient symbolic solutions, which rarely involve systematic structures or redundant elements that are not crucial for solving the task (see, e.g., Galantucci, 2005; Fay et al., 2013; cf. Nölle et al., 2018; Raviv et al., 2019). By contrast, transmission creates biases for simplicity and learnability. Variants are more likely to get passed on if they are easier to remember. Participants in iterated learning studies therefore favour compressible analytic structures that are easier to learn over simple holistic signals, which creates a bottleneck for compositional structure to pass through (Kirby et al., 2008). However, the most learnable systems are not necessarily very expressive and useful for communication (e.g., a single label that applies to all available meanings). Newer studies have therefore begun to combine interaction and transmission paradigms by using chains of dyads and consistently shown that this results in languages that are both easy to learn and transmit as well as expressive and efficient for communication (Kirby et al., 2015; Motamedi et al., 2019).

Other paradigms have been added, such as replacement and community paradigms that allow studying how symbol variants evolve as they diffuse in a population, and how local usage events and changes can lead to the emergence of global conventionalised patterns (Fay et al., 2010; Galantucci, Theisen, et al., 2012; Fay \& Ellison, 2013). In this context, ES can be used to study the relationship between language and its wider environment: Raviv and colleagues (2019) tested a correlation that has been found between grammatical complexity and population size in spoken and signed languages (Lupyan \& Dale, 2010; Meir et al., 2012). They had participants interact in micro-societies with four or eight members in fully connected networks (see Figures 1e,g) and found that larger communities developed more systematic lan guages and did so faster, which corroborates the patterns among natural languages. 


\section{Box 1: Summary of the Experimental Semiotics approach}

To illustrate how ES studies give rise to a novel communication system in the lab, Figure 2 provides an overview of common components of ES experiments: Each sign system ultimately derives from form-meaning mappings. The signalling space (Fig. 2a top) determines the form, i.e. which communication channel is chosen. The modality of the channel (e.g., gesture, vocalisation, drawing) provides affordances for iconicity and determines how signals can be motivated. For example, in Fay et al.'s (2007) drawing study, participants improvised iconic drawings of concept such as computer screen (bottom). This allows grounding communicative signals and enables a process of conventionalisation where, due to the interactive nature of the game, gradual symbolification turns icons into arbitrary symbols. The meaning space (Fig. 2b) determines the semantic content the signals are mapped onto, i.e. what is talked about. This could, e.g., be the location of four rooms with corresponding symbols, such as in Galantucci's (2005) original coordination game (left) or a set of cartoon pictures with systematic elements such as in Nölle et al.'s (2018) silent gesture study (right). In most cases the sign inventory (or lexicon of an artificial language) will come to reflect the referential targets that have to be communicated. The wider task environment (Fig. 2c) can exert further pressures by highlighting salient properties or making aspects of the referential environment (ir-)relevant. This can include aspects of where the experiment is situated, like different layouts in the Maze Game (top) that make specific ways of describing locations particularly salient (Garrod \& Anderson, 1987; Nölle, Fusaroli, et al., 2020), or the presence of features in the referential context itself: If referential targets include different shapes (centre left), each signal is likely to be mapped onto a shape, however, if both shape and colour are relevant meaning dimensions (centre right), then the communication system will likely encode both (Silvey et al., 2015; Winters et al., 2015). Furthermore, aspects of the communicative environment itself can shape sign systems (bottom left): Referring to visible objects on a table is vastly different from communicating things that are displaced from the communicative context (e.g., shown briefly on a screen before communication takes place), since strategies like pointing are disabled. Lastly, the social environment, such as community size or network structure can shape aspects such as complexity of the sign system or the diffusion of variants (Fay \& Ellison, 2013; Raviv et al., 2019, 2020). The paradigm that is chosen for an ES experiment is tightly linked to task dynamics. For example, artificial language learning is usually used when studying vertical transmission (Fig. 2e, left), like in iterated learning designs (Kirby et al., 2014). By contrast, communication studies use referential tasks or coordination games to study horizontal interaction. For example, Roberts and Galantucci (2012) had dyads communicate targets from a set of referents placed on a grid via a track pad for graphical communication (Fig. 2e, right). The task dynamics (Fig. 2d), in turn, then shape the characteristics of the communication system in different ways: Transmission puts pressure on systems to become simpler, more compressible and easier to learn, whereas interaction puts pressure on signals to become more efficient over time, and makes them informative and expressive in the given context (i.e. satisfy the meaning space and be functionally adaptive to the environment). These pressures are necessary to give rise to design features such as systematicity or duality of patterning (see section 3.2). For more details on variations of task dynamics see also Figure 1 above. A final set of constraints comes from participants' biological and cognitive biases (Fig. 2f). This involves, e.g., innate learning biases that are amplified over transmission and lead to regularity (Kirby et al., 2007). Additionally, linguistic background should be controlled for depending on the phenomena of interest: E.g., when studying the emergence of noun phrase word order with English speakers, it makes sense to use a post-nominal language to preclude native biases, and silent gesture experiments should not be conducted with fluent signers who are more experienced in the manual modality. Lastly, the cultural background and cognitive style might affect the pre-established grammatical or conceptual categories (e.g., colour, space) that participants will impose on the task, which can lead to different outcomes. ES studies therefore try to control such variables and/or study samples from different populations for a cross-cultural perspective that gives insights into universals and variation of human semiotic behaviour.

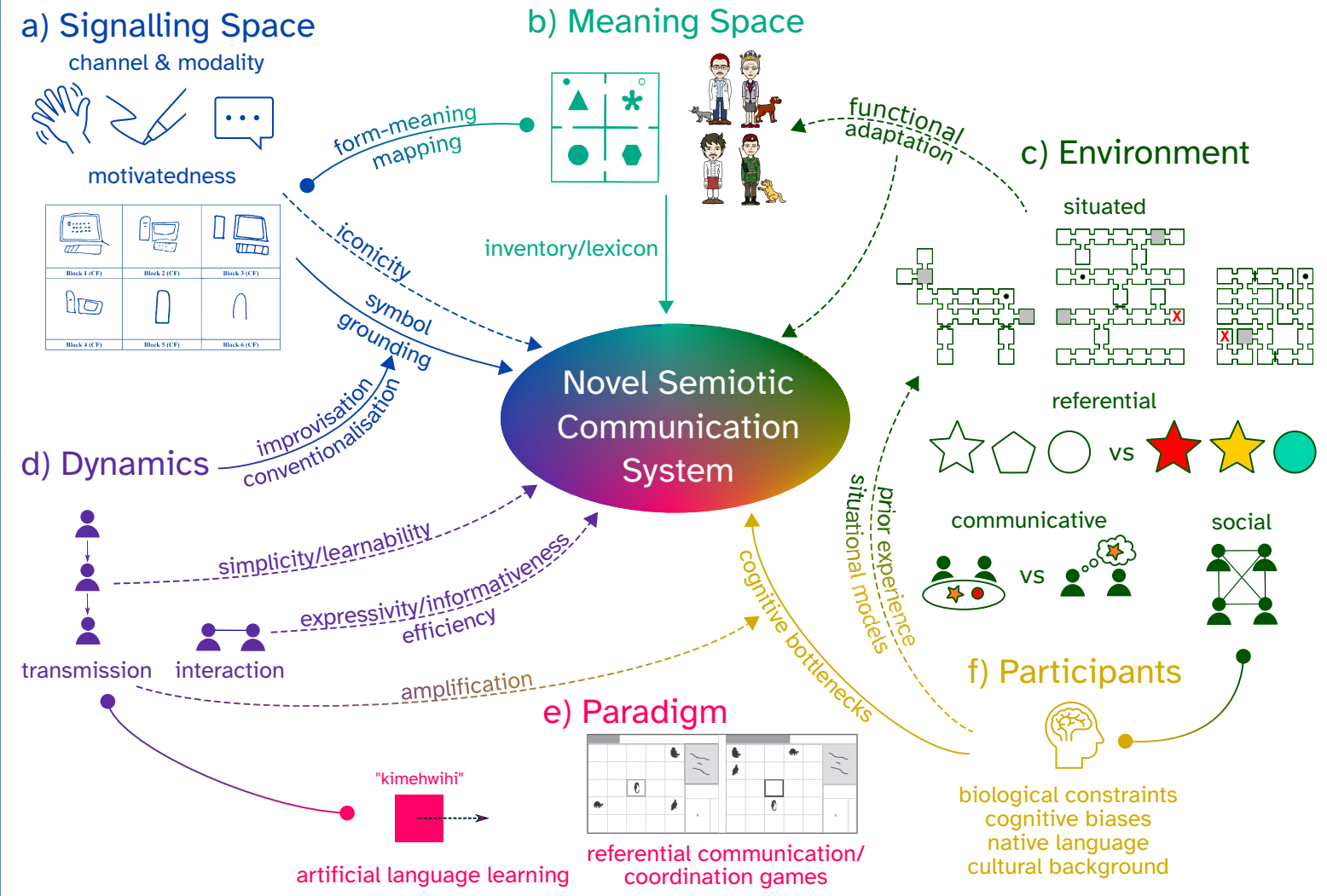

Figure 2: Components of Experimental Semiotics studies that give rise to a novel semiotic communication system. Solid lines indicate processes and mechanisms in the evolution of a sign system, dashed lines indicate pressures shaping its core properties. 


\section{Open challenges and future directions}

\subsection{Limitations}

Although ES has delivered many of its promises and proven to be a powerful engine of discovery for the cognitive, language, and social sciences, it does not come without limitations. While ES has successfully modelled mechanisms underlying the basic building blocks of communication, we are still far from observing the emergence of full-fledged 'languages', in the sense of fully productive, flexible and open-ended communication systems. This is in part because artificial communication systems are often miniature versions of languages consisting of small inventories of form-meaning mappings that can only be applied in the restricted context of the task itself. Additionally, ES has focused on isolating the core properties of language and has only more recently be integrated with research into more concrete phenomena: For example, after the success of ES paradigms in uncovering cognitive biases and mechanisms shaping communication systems, artificial language learning experiments are now used more routinely to investigate the origins of typological patterns such as word order universals (Culbertson et al., 2012, 2020), animacy markers (Vihman et al., 2018) or person systems (Maldonado \& Culbertson, 2020). Furthermore, the general forces that shape communication systems in ES experiments, such as pressures for expressivity/informativeness and simplicity/learnability have - beyond lab experiments - been confirmed to shape features of natural languages, such as semantic category system, e.g., to express kinship (Kemp \& Regier, 2012), or colours (Gibson et al., 2019). ES has also been applied to subfields such as sociolinguistics, simulating the formation of dialects (Sneller \& Roberts, 2018), to phonology (Roberts \& Clark, 2020), and to experimental pragmatics (Tinits et al., 2017; Rubio-Fernandez \& Jara-Ettinger, 2020). Roberts and Sneller (2020) therefore view ES as part of a newly "integrated science of language evolution" that allows studying both the emergence of structure and its ongoing change and variation (see also Roberts, 2017; Hartmann, 2020 on the distinction between language evolution and language change). For the near future, the challenge remains for ES to demonstrate that it cannot only serve as a tool for simulating the emergence of simple task-oriented communication systems but also help explain the full richness and complexity of natural language.

Tamariz (2017, p. 402) identified several 'future issues' for the field. Some of these have been advanced in recent years, like integrating ES more with other disciplines as described above, or the manipulation of population size and structure in experiments, which have since been conducted (see section 4.2). However, other challenges remain: The differential contribution of children and adults to the emergence of language is still poorly understood, and only few studies have begun to address this question (Kempe et al., 2015, 2019; Raviv \& Arnon, 2018).

Another challenge lies in the use of more 'naturalistic games' (Tamariz, 2017, p. 403), which pertains to the ecological validity of ES experiments. To get humans, who already speak or sign a language, to create novel forms of communication in the lab, researchers have conceived elegant, but ultimately highly artificial settings that hardly resemble real communicative interactions, which in turn renders unclear whether the results are representative of and generalisable to real-world phenomena. For a long time, it has virtually been agreed upon that ecological validity is something that needs to be sacrificed to gain experimental control (e.g., to isolate the causal effect of a variable). However, as Peeters (2019) points out, these two aspects of experimental design are actually orthogonal properties rather than the ends of a continuum. This becomes clear when considering recent advances in technology, such as immersive virtual reality (VR). Using VR, it is possible to design immersive and realistic communication tasks that still allow for full experimental control of all variables. This has for instance be used to study how spatial language adapts to the environment (Nölle, Kirby, et al., 2020) and could easily be extended to other multimodal communication tasks. Similarly, smartphone apps now 
offer a novel opportunity for studying the emergence and diffusion of communication systems in large populations for which computational models were previously required (Morin et al., $2018,2020)$. We thus believe that several limitations of ES can be overcome as novel technologies and the integration of ES paradigms into other disciplines will greatly expand what the approach is capable of.

\subsection{Integration of ES and neuroscience in a neurosemiotic framework}

While ES has been successfully applied in the language and psychological sciences, other fields still bear a lot of potential for cross-fertilisation and a tighter integration of ES methods. In neuroscience, recent years have seen calls for a tighter integration with the social sciences and application of neuroscientific findings (Ibáñez et al., 2017), for instance to better understand how social processes such as value-based decision making, influence and persuasion (Falk \& Scholz, 2018), or social cognitive abilities like mentalising and Theory of Mind (Frith \& Frith, 2012) are actually embedded in situated social interactions.

As we have seen, ES allows studying human meaning-making in situated and social settings (see Box 1, Fig. 2c), in which interlocutors must rely on exactly these social processes embedded in joint actions to create shared symbols and coordinate successfully (e.g., Clark, 1996). Building common ground and establishing form-meaning mappings based on feedback from the interlocutor and environment requires joint attention, perspective-taking and mentalising abilities, and even so, some individual participants still fail at solving ES tasks successfully (Galantucci, 2005). This offers the perfect opportunity to study the underlying social and affective neural processes under 'extreme' conditions and, with advances in paradigms and technology (such as VR), in naturalistic but controlled settings.

In the past, neuroscience has mostly focussed on studying cognitive processes in individual brains (Hasson et al., 2012). One way to study social phenomena with neuroscientific methods is the social observation approach, where participants in the brain scanner passively encounter social stimuli. More recently, some studies have explicitly looked at symbolic behaviour. For instance, Rocca et al. (2020) used contextualised dialogical narratives in the fMRI scanner to show that spatial demonstratives ("this", "that"), which are used to establish joint attention in interaction, rely on neural resources for perception, attention and extra-linguistic cognition. Tylén et al. (2016) combined a behavioural task with a brain scan to show how symbolic artefacts engage social brain areas. Participants had to collective build symbolic representations of abstract concepts using LEGO bricks. Viewing photographs of the models was associated with social areas in the brain, and more interestingly contrasting activation for viewing one's own models or those created by other groups, suggesting that neural activity can capture a perceiver's attitude towards a cultural symbol.

A new 'second-person' neuroscience tries to go even further by directly studying brain-tobrain couplings with a 'dual-brain approach' or 'hyperscanning' (Montague, 2002), where two participants in separate fMRI scanners interact directly with each other (Redcay \& Schilbach, 2019). Such an approach allows measuring coupling (synchronous activity in the same regions of both brains) as well as inter-brain connectivity (brain activity in one participant that correlates with brain activity in a distinct area in another participant) in direct social interaction, and has demonstrated how the brains of two interlocutors are coupled/misaligned during successful/failed communication using speech (Stephens et al., 2010) or gesture (Schippers et al., 2010). This dual-brain approach allows studying social cognitive processes such as mentalizing, joint attention, reciprocal interaction and group dynamics (see Redcay \& Schilbach, 2019 for a review).

Combining ES with this second-person neuroscience approach will allow investigating the brain-to-brain coupling processes involved in symbol grounding, how this neural coupling emerges gradually as common ground is established between participants, and to what extent 
it is required for successful communication. Some initial studies in this direction have already been conducted: Stolk et al. (2014) used transcranial magnetic stimulation during an ES-style communication task to show which areas are necessary for action understanding when establishing communication. In this vein, the combination of ES and modern neuroscience techniques allows deriving both a behavioural and neural picture of how people establish conceptual alignment (Pickering \& Garrod, 2004; Stolk et al., 2016) and resolve ambiguity, uncertainty and asymmetries to successfully communicate (Stolk et al., 2020; see also the article by Stolk, Toni and Basnakova in this volume).

As part of an integrated neurosemiotics that views human meaning-making as both socially and biologically grounded (see Deacon, 1997; Plebe \& Cruz, 2016 and other articles in this volume), ES can not only inspire neuroscience to integrate more socially focused interaction paradigms, but likewise benefit from the use of neuroscientific methods. Behavioural data can provide only a sketchy picture of the cognitive, emotional and social processes involved in ES experiments, and people are notoriously unreliable in describing them at a subjective level. Here, both neural data (fMRI, MEG, EEG) as well as biological markers and physiological measures (heart rate, skin conductance) can give insights into the neurobiological underpinnings of embodied synchronisations and interpersonal synergies that are necessary to achieve meaning-making, but have mostly been studied on a behavioural level (Louwerse et al., 2012; Fusaroli \& Tylén, 2016).

\section{Conclusions}

Taken together, the first decade of ES research established it as a serious approach for studying human semiosis and the evolution of communication systems in the lab using direct interaction under controlled conditions (Galantucci, Garrod, et al., 2012). During its second decade, ES has proven to be a reliable tool that can be applied in various disciplines and fields to study the full range of human semiosis. It is our hope that, in its third decade, ES will improve upon some of its limitations. This includes more naturalistic tasks and a tighter integration of neurological and biological perspectives, to understand not only how humans are able to create sign systems at the behavioural level but also which neurobiological processes are underlying meaning-making in these contexts. ES can thus serve as an important set of methods in a modern neurosemiotic framework that approaches human semiosis in its natural ecology, namely goaloriented face-to-face interaction between embodied agents situated in an environment.

\section{References}

Bartlett, F. C. (1932). Remembering. A study in experimental and social psychology. Cambridge University Press.

Bavelas, A. (1950). Communication patterns in task-oriented groups. The Journal of the Acoustical Society of America, 22(6), 725-730.

Bavelas, A. (1952). Communication patterns in problem-solving groups. In H. von Foerster (Ed.), Cybernetics. Circularcausal and feedback mechanisms in biological and social systems. Transactions of the ninth conference (pp. 1-44). Josiah Macy Jr. Foundation New York, NY.

Brighton, H. (2002). Compositional Syntax From Cultural Transmission. Artificial Life, 8(1), 25-54. https://doi.org/10.1162/106454602753694756

Brighton, H., Smith, K., \& Kirby, S. (2005). Language as an evolutionary system. Physics of Life Reviews, 2(3), 177-226. https://doi.org/10.1016/j.plrev.2005.06.001 
Carr, J. W., Smith, K., Cornish, H., \& Kirby, S. (2017). The Cultural Evolution of Structured Languages in an Open-Ended, Continuous World. Cognitive Science, 41(4), 892-923. https://doi.org/10.1111/cogs. 12371

Cassirer, E. (1953). The philosophy of symbolic forms. Vol. I: Language (pp. xiv, 328). Yale University Press.

Christiansen, M. H., \& Chater, N. (2008). Language as shaped by the brain. Behavioral and Brain Sciences, 31(5), 489-509. https://doi.org/10.1017/S0140525X08004998

Christiansen, M. H., \& Kirby, S. (2003). Language Evolution: The Hardest Problem in Science. In M. H. Christiansen \& S. Kirby (Eds.), Language Evolution (pp. 1-15). Oxford University Press.

Clark, H. H. (1996). Using Language. Cambridge University Press.

Clark, H. H., \& Wilkes-Gibbs, D. (1986). Referring as a collaborative process. Cognition, 22(1), 1-39. https://doi.org/10.1016/0010-0277(86)90010-7

Culbertson, J., Schouwstra, M., \& Kirby, S. (2020). From the world to word order: Deriving biases in noun phrase order from statistical properties of the world. Language, 96(3), 696717. https://doi.org/10.1353/lan.2020.0045

Culbertson, J., Smolensky, P., \& Legendre, G. (2012). Learning biases predict a word order universal. Cognition, 122(3), 306-329. https://doi.org/10.1016/j.cognition.2011.10.017

de Saussure, F. (1916). Cours de Linguistique Générale. Payot.

Deacon, T. W. (1997). The Symbolic Species: The Co-Evolution of Language and the Brain. Norton.

Dingemanse, M., Blasi, D. E., Lupyan, G., Christiansen, M. H., \& Monaghan, P. (2015). Arbitrariness, Iconicity, and Systematicity in Language. Trends in Cognitive Sciences, 19(10), 603-615. https://doi.org/10.1016/j.tics.2015.07.013

Eryilmaz, K., \& Little, H. (2017). Using leap motion to investigate the emergence of structure in speech and language. Behavior Research Methods, 49(5), 1748-1768. https://doi.org/10.3758/s13428-016-0818-x

Esper, E. A. (1925). A technique for the experimental investigation of associative interference in artificial linguistic material. Linguistic Society of America.

Falk, E., \& Scholz, C. (2018). Persuasion, Influence, and Value: Perspectives from Communication and Social Neuroscience. Annual Review of Psychology, 69(1), 329-356. https://doi.org/10.1146/annurev-psych-122216-011821

Fay, N., Arbib, M., \& Garrod, S. (2013). How to Bootstrap a Human Communication System. Cognitive Science, 37(7), 1356-1367. https://doi.org/10.1111/cogs.12048

Fay, N., \& Ellison, T. M. (2013). The Cultural Evolution of Human Communication Systems in Different Sized Populations: Usability Trumps Learnability. PLoS ONE, 8(8), e 71781. https://doi.org/10.1371/journal.pone.0071781

Fay, N., Garrod, S., Roberts, L., \& Swoboda, N. (2010). The Interactive Evolution of Human Communication Systems. Cognitive Science, 34(3), 351-386. https://doi.org/10.1111/j.1551-6709.2009.01090.x

Fay, N., Lister, C. J., Mark Ellison, T., \& Goldin-Meadow, S. (2014). Creating a communication system from scratch: Gesture beats vocalization hands down. Frontiers in Psychology, 5(APR), 1-12. https://doi.org/10.3389/fpsyg.2014.00354

Fehér, O., Wang, H., Saar, S., Mitra, P. P., \& Tchernichovski, O. (2009). De novo establishment of wild-type song culture in the zebra finch. Nature, 459(7246), 564-568. https://doi.org/10.1038/nature07994

Fitch, W. T. (2010). The Evolution of Language. Cambridge University Press.

Fitch, W. T. (2017). Empirical approaches to the study of language evolution. Psychonomic Bulletin \& Review, 24(1), 3-33. https://doi.org/10.3758/s13423-017-1236-5 
Frank, M. C., \& Goodman, N. D. (2012). Predicting Pragmatic Reasoning in Language Games. Science, 336(6084), 998-998. https://doi.org/10.1126/science.1218633

Frith, C. D., \& Frith, U. (2012). Mechanisms of Social Cognition. Annual Review of Psychology, 63, 287-313.

Fusaroli, R., \& Tylén, K. (2016). Investigating Conversational Dynamics: Interactive Alignment, Interpersonal Synergy, and Collective Task Performance. Cognitive Science, 40(1), 145-171. https://doi.org/10.1111/cogs.12251

Fusellier-Souza, I. (2006). Emergence and Development of Signed Languages. Sign Language Studies, 7(1), 30-56. JSTOR.

Galantucci, B. (2005). An Experimental Study of the Emergence of Human Communication $\begin{array}{lll}\text { Systems. } & \text { Cognitive } & \text { Science, }\end{array}$ https://doi.org/10.1207/s15516709cog0000_34

Galantucci, B. (2017). Experimental Semiotics. In B. Galantucci, Oxford Research Encyclopedia of Linguistics. Oxford University Press. https://doi.org/10.1093/acrefore/9780199384655.013.210

Galantucci, B., Garrod, S., \& Roberts, G. (2012). Experimental Semiotics. Language and Linguistics Compass, 6(8), 477-493. https://doi.org/10.1002/lnc3.351

Galantucci, B., Kroos, C., \& Rhodes, T. (2010). The effects of rapidity of fading on communication systems. Interaction Studies, 11(1), 100-111. https://doi.org/10.1075/is.11.1.03gal

Galantucci, B., \& Roberts, G. (2012). EXPERIMENTAL SEMIOTICS: AN ENGINE OF DISCOVERY FOR UNDERSTANDING HUMAN COMMUNICATION. Advances in Complex Systems, 15(03n04), 1150026. https://doi.org/10.1142/S0219525911500263

Galantucci, B., Theisen, C., Gutierrez, E. D., Kroos, C., \& Rhodes, T. (2012). The diffusion of novel signs beyond the dyad. Language Sciences, 34(5), 583-590. https://doi.org/10.1016/j.langsci.2012.03.013

Garrod, S., \& Anderson, A. (1987). Saying what you mean in dialogue: A study in conceptual and semantic co-ordination. Cognition, 27(2), 181-218. https://doi.org/10.1016/00100277(87)90018-7

Garrod, S., \& Doherty, G. (1994). Conversation, co-ordination and convention: An empirical investigation of how groups establish linguistic conventions. Cognition, 53(3), 181-215. https://doi.org/10.1016/0010-0277(94)90048-5

Garrod, S., Fay, N., Lee, J., Oberlander, J., \& MacLeod, T. (2007). Foundations of Representation: Where Might Graphical Symbol Systems Come From? Cognitive Science, 31(6), 961-987. https://doi.org/10.1080/03640210701703659

Garrod, S., \& Pickering, M. J. (2009). Joint Action, Interactive Alignment, and Dialog. Topics in Cognitive Science, 1(2), 292-304. https://doi.org/10.1111/j.1756-8765.2009.01020.x

Gibson, E., Futrell, R., Piantadosi, S. P., Dautriche, I., Mahowald, K., Bergen, L., \& Levy, R. (2019). How Efficiency Shapes Human Language. Trends in Cognitive Sciences, 23(5), 389-407. https://doi.org/10.1016/j.tics.2019.02.003

Goldin-Meadow, S., So, W. C., Özyürek, A., \& Mylander, C. (2008). The natural order of events: How speakers of different languages represent events nonverbally. Proceedings of the National Academy of Sciences, 105(27), 9163-9168. https://doi.org/10.1073/pnas.0710060105

Goodman, N. D., \& Frank, M. C. (2016). Pragmatic Language Interpretation as Probabilistic Inference. Trends in Cognitive Sciences, 20(11), 818-829. https://doi.org/10.1016/j.tics.2016.08.005

Grice, H. P. (1975). Logic and conversation. In P. Cole \& J. L. Morgan (Eds.), Syntax and semantics, vol. III (pp. 183-198). Academic Press. 
Griffiths, T. L., \& Kalish, M. L. (2007). Language Evolution by Iterated Learning With Bayesian Agents. Cognitive Science, 31(3), 441-480. https://doi.org/10.1080/15326900701326576

Harnad, S. (1990). The symbol grounding problem. Physica D: Nonlinear Phenomena, 42(1), 335-346. https://doi.org/10.1016/0167-2789(90)90087-6

Hartmann, S. (2020). Language change and language evolution: Cousins, siblings, twins? Glottotheory, 11(1), 15-39. https://doi.org/10.1515/glot-2020-2003

Hasson, U., Ghazanfar, A. A., Galantucci, B., Garrod, S., \& Keysers, C. (2012). Brain-to-brain coupling: A mechanism for creating and sharing a social world. Trends in Cognitive Sciences, 16(2), 114-121. https://doi.org/10.1016/j.tics.2011.12.007

Healey, P. G. T. (2008). Interactive Misalignment: The Role of Repair in the Development of Group Sub-languages. In R. Cooper \& R. Kempson (Eds.), Language in Flux (pp. 13-39). Palgrave Macmillan.

Hockett, C. F. (1960). The Origin of Speech. Scientific American, 203(3), 88-97. https://doi.org/10.2307/24940617

Holler, J., \& Levinson, S. C. (2019). Multimodal Language Processing in Human Communication. Trends in Cognitive Sciences, 23(8), 639-652. https://doi.org/10.1016/j.tics.2019.05.006

Horton, W. S., \& Keysar, B. (1996). When do speakers take into account common ground? Cognition, 59(1), 91-117. https://doi.org/10.1016/0010-0277(96)81418-1

Ibáñez, A., Sedeño, L., \& García, A. M. (2017). Neuroscience and Social Science: The Missing Link. Springer.

Kemp, C., \& Regier, T. (2012). Kinship Categories Across Languages Reflect General Communicative Principles. Science, 336(6084), 1049-1054. https://doi.org/10.1126/science. 1218811

Kempe, V., Gauvrit, N., \& Forsyth, D. (2015). Structure emerges faster during cultural transmission in children than in adults. Cognition, 136, 247-254. https://doi.org/10.1016/j.cognition.2014.11.038

Kempe, V., Gauvrit, N., Gibson, A., \& Jamieson, M. (2019). Adults are more efficient in creating and transmitting novel signalling systems than children. Journal of Language Evolution, 4(1), 44-70. https://doi.org/10.1093/jole/lzy012

Kirby, S. (2017). Culture and biology in the origins of linguistic structure. Psychonomic Bulletin \& Review, 24(1), 118-137. https://doi.org/10.3758/s13423-016-1166-7

Kirby, S., Cornish, H., \& Smith, K. (2008). Cumulative cultural evolution in the laboratory: An experimental approach to the origins of structure in human language. Proceedings of the National Academy of Sciences, 105(31), 10681-10686. https://doi.org/10.1073/pnas.0707835105

Kirby, S., Dowman, M., \& Griffiths, T. L. (2007). Innateness and culture in the evolution of language. Proceedings of the National Academy of Sciences, 104(12), 5241-5245. https://doi.org/10.1073/pnas.0608222104

Kirby, S., Griffiths, T., \& Smith, K. (2014). Iterated learning and the evolution of language. Current Opinion in Neurobiology, 28, 108-114. https://doi.org/10.1016/j.conb.2014.07.014

Kirby, S., \& Hurford, J. R. (2002). The Emergence of Linguistic Structure: An Overview of the Iterated Learning Model. In A. Cangelosi \& D. Parisi (Eds.), Simulating the Evolution of Language (pp. 121-147). Springer. https://doi.org/10.1007/978-1-4471-0663-0_6

Kirby, S., Tamariz, M., Cornish, H., \& Smith, K. (2015). Compression and communication in the cultural evolution of linguistic structure. Cognition, 141, 87-102. https://doi.org/10.1016/j.cognition.2015.03.016 
Kocab, A., Pyers, J., \& Senghas, A. (2015). Referential shift in Nicaraguan Sign Language: A transition from lexical to spatial devices. Frontiers in Psychology, 5. https://doi.org/10.3389/fpsyg.2014.01540

Krauss, R. M., \& Weinheimer, S. (1964). Changes in reference phrases as a function of frequency of usage in social interaction: A preliminary study. Psychonomic Science, 1(1), 113-114. https://doi.org/10.3758/BF03342817

Little, H., Eryılmaz, K., \& de Boer, B. (2017). Signal dimensionality and the emergence of combinatorial structure. Cognition, 168, 1-15. https://doi.org/10.1016/j.cognition.2017.06.011

Louwerse, M. M., Dale, R., Bard, E. G., \& Jeuniaux, P. (2012). Behavior Matching in Multimodal Communication Is Synchronized. Cognitive Science, 36(8), 1404-1426. https://doi.org/10.1111/j.1551-6709.2012.01269.x

Lupyan, G., \& Dale, R. (2010). Language Structure Is Partly Determined by Social Structure. PLOS ONE, 5(1), e8559. https://doi.org/10.1371/journal.pone.0008559

Maldonado, M., \& Culbertson, J. (2020). Person of Interest: Experimental Investigations into the Learnability of Person Systems. Linguistic Inquiry, 1-42. https://doi.org/10.1162/ling_a_00406

Meir, I., Israel, A., Sandler, W., Padden, C. A., \& Aronoff, M. (2012). The influence of community on language structure: Evidence from two young sign languages. Linguistic Variation, 12(2), 247-291. https://doi.org/10.1075/lv.12.2.04mei

Montague, P. (2002). Hyperscanning: Simultaneous fMRI during Linked Social Interactions. NeuroImage, 16(4), 1159-1164. https://doi.org/10.1006/nimg.2002.1150

Morin, O., Winters, J., Müller, T. F., Morisseau, T., Etter, C., \& Greenhill, S. J. (2018). What smartphone apps may contribute to language evolution research. Journal of Language Evolution, 3(2), 91-93. https://doi.org/10.1093/jole/lzy005

Morin, O., Winters, J., Müller, T., \& Morisseau, T. (2020). An overview of the" Color Game" App project.

Motamedi, Y., Schouwstra, M., Smith, K., Culbertson, J., \& Kirby, S. (2019). Evolving artificial sign languages in the lab: From improvised gesture to systematic sign. Cognition, 192, 103964. https://doi.org/10.1016/j.cognition.2019.05.001

Motamedi, Y., Smith, K., Schouwstra, M., Culbertson, J., \& Kirby, S. (2018). The emergence of systematic argument distinctions in artificial sign languages. PsyArXiv. https://doi.org/10.31234/osf.io/p6zy4

Nölle, J., Fusaroli, R., Mills, G. J., \& Tylén, K. (2020). Language as shaped by the environment: Linguistic construal in a collaborative spatial task. Palgrave Communications, 6(1), 1-10. https://doi.org/10.1057/s41599-020-0404-9

Nölle, J., Hartmann, S., \& Tinits, P. (2020). Language evolution research in the year 2020: A survey of new directions. Language Dynamics and Change, 10(1), 3-26. https://doi.org/10.1163/22105832-bja10005

Nölle, J., Kirby, S., Culbertson, J., \& Smith, K. (2020). Does environment shape spatial language? A virual reality approach. In A. Ravignani, C. Barbieri, M. Martins, M. Flaherty, Y. Jadoul, E. Lattenkamp, H. Little, K. Mudd, \& T. Verhoef (Eds.), The evolution of language: Proceedings of the 13th international conference (EvoLang13) (pp. 321-323).

Nölle, J., Staib, M., Fusaroli, R., \& Tylén, K. (2018). The emergence of systematicity: How environmental and communicative factors shape a novel communication system. Cognition, 181, 93-104. https://doi.org/10.1016/j.cognition.2018.08.014

Noveck, I. A., \& Sperber, D. (Eds.). (2004). Experimental pragmatics. Palgrave Macmillan.

Ortega, G., \& Özyürek, A. (2020). Systematic mappings between semantic categories and types of iconic representations in the manual modality: A normed database of silent gesture. Behavior Research Methods, 52(1), 51-67. https://doi.org/10.3758/s13428-019-01204-6 
Peeters, D. (2019). Virtual reality: A game-changing method for the language sciences. Psychonomic Bulletin \& Review. https://doi.org/10.3758/s13423-019-01571-3

Perlman, M., Dale, R., \& Lupyan, G. (2015). Iconicity can ground the creation of vocal symbols. Royal Society Open Science, 2(8), 150152. https://doi.org/10.1098/rsos.150152

Perlman, M., \& Lupyan, G. (2018). People Can Create Iconic Vocalizations to Communicate Various Meanings to Naïve Listeners. Scientific Reports, 8(1), 2634. https://doi.org/10.1038/s41598-018-20961-6

Perniss, P. (2018). Why We Should Study Multimodal Language. Frontiers in Psychology, 9. https://doi.org/10.3389/fpsyg.2018.01109

Pickering, M. J., \& Garrod, S. (2004). Toward a mechanistic psychology of dialogue. Behavioral and Brain Sciences, 27(02). https://doi.org/10.1017/S0140525X04000056

Plebe, A., \& Cruz, V. M. D. L. (2016). Neurosemantics: Neural Processes and the Construction of Linguistic Meaning. Springer.

Raviv, L., \& Arnon, I. (2018). Systematicity, but not compositionality: Examining the emergence of linguistic structure in children and adults using iterated learning. Cognition, 181, 160-173. https://doi.org/10.1016/j.cognition.2018.08.011

Raviv, L., Meyer, A., \& Lev-Ari, S. (2019). Compositional structure can emerge without generational transmission. Cognition, 182, 151-164. https://doi.org/10.1016/j.cognition.2018.09.010

Raviv, L., Meyer, A., \& Lev-Ari, S. (2020). The Role of Social Network Structure in the Emergence of Linguistic Structure. Cognitive Science, 44(8), e12876. https://doi.org/10.1111/cogs.12876

Redcay, E., \& Schilbach, L. (2019). Using second-person neuroscience to elucidate the mechanisms of social interaction. Nature Reviews Neuroscience, 20(8), 495-505. https://doi.org/10.1038/s41583-019-0179-4

Roberts, G. (2017). The linguist's Drosophila: Experiments in language change. Linguistics Vanguard, 3(1). https://doi.org/10.1515/lingvan-2016-0086

Roberts, G., \& Clark, R. (2020). Dispersion, communication, and alignment: An experimental study of the emergence of structure in combinatorial phonology. Journal of Language Evolution, 5(2), 121-139. https://doi.org/10.1093/jole/lzaa004

Roberts, G., \& Galantucci, B. (2012). The emergence of duality of patterning: Insights from the laboratory. Language and Cognition, 4(04), 297-318. https://doi.org/10.1515/langcog2012-0017

Roberts, G., Lewandowski, J., \& Galantucci, B. (2015). How communication changes when we cannot mime the world: Experimental evidence for the effect of iconicity on combinatoriality. Cognition, 141, 52-66. https://doi.org/10.1016/j.cognition.2015.04.001

Roberts, G., \& Sneller, B. (2020). Empirical foundations for an integrated study of language evolution. Language Dynamics and Change, 10(2), 188-229. https://doi.org/10.1163/22105832-bja10001

Rocca, R., Coventry, K. R., Tylén, K., Staib, M., Lund, T. E., \& Wallentin, M. (2020). Language beyond the language system: Dorsal visuospatial pathways support processing of demonstratives and spatial language during naturalistic fast fMRI. NeuroImage, 216, 116128. https://doi.org/10.1016/j.neuroimage.2019.116128

Rubio-Fernandez, P., \& Jara-Ettinger, J. (2020). Incrementality and efficiency shape pragmatics across languages. Proceedings of the National Academy of Sciences, 117(24), 1339913404. https://doi.org/10.1073/pnas.1922067117

Ruiter, J. P. de, Noordzij, M. L., Newman-Norlund, S., Newman-Norlund, R., Hagoort, P., Levinson, S. C., \& Toni, I. (2010). Exploring the cognitive infrastructure of communication. Interaction Studies, 11(1), 51-77. https://doi.org/10.1075/is.11.1.05rui 
Sandler, W., Aronoff, M., Meir, I., \& Padden, C. (2011). The gradual emergence of phonological form in a new language. Natural Language \& Linguistic Theory, 29(2), 503-543. https://doi.org/10.1007/s11049-011-9128-2

Schippers, M. B., Roebroeck, A., Renken, R., Nanetti, L., \& Keysers, C. (2010). Mapping the information flow from one brain to another during gestural communication. Proceedings of the National Academy of Sciences, 107(20), 9388-9393. https://doi.org/10.1073/pnas.1001791107

Schouwstra, M., \& de Swart, H. (2014). The semantic origins of word order. Cognition, 131(3), 431-436. https://doi.org/10.1016/j.cognition.2014.03.004

Scott-Phillips, T. C., Kirby, S., \& Ritchie, G. R. S. (2009). Signalling signalhood and the emergence of communication. Cognition, 113(2), 226-233. https://doi.org/10.1016/j.cognition.2009.08.009

Selten, R., \& Warglien, M. (2007). The emergence of simple languages in an experimental coordination game. Proceedings of the National Academy of Sciences, 104(18), 73617366. https://doi.org/10.1073/pnas.0702077104

Senghas, A. (2004). Children Creating Core Properties of Language: Evidence from an Emerging Sign Language in Nicaragua. Science, 305(5691), 1779-1782. https://doi.org/10.1126/science.1100199

Silvey, C., Kirby, S., \& Smith, K. (2015). Word Meanings Evolve to Selectively Preserve Distinctions on Salient Dimensions. Cognitive Science, 39(1), 212-226. https://doi.org/10.1111/cogs. 12150

Smith, K., Perfors, A., Fehér, O., Samara, A., Swoboda, K., \& Wonnacott, E. (2017). Language learning, language use and the evolution of linguistic variation. Philosophical Transactions of the Royal Society B: Biological Sciences, 372(1711), 20160051. https://doi.org/10.1098/rstb.2016.0051

Smith, K., Tamariz, M., \& Kirby, S. (2013). Linguistic Structure is an Evolutionary Trade-Off between Simplicity and Expressivity. In M. Knauff, M. Pauen, N. Sebanz, \& I. Wachsmuth (Eds.), Cooperative Minds: Social Interaction and Group Dynamics (pp. 1348-1353). Cognitive Science Society.

Sneller, B., \& Roberts, G. (2018). Why some behaviors spread while others don't: A laboratory simulation of dialect contact. Cognition, 170, 298-311. https://doi.org/10.1016/j.cognition.2017.10.014

Steels, L. (2011). Modeling the cultural evolution of language. Physics of Life Reviews, 8(4), 339-356. https://doi.org/10.1016/j.plrev.2011.10.014

Stephens, G. J., Silbert, L. J., \& Hasson, U. (2010). Speaker-listener neural coupling underlies successful communication. Proceedings of the National Academy of Sciences, 107(32), 14425-14430. https://doi.org/10.1073/pnas.1008662107

Stolk, A., Bašnáková, J., \& Toni, I. (2020). Joint epistemic engineering: The neglected process of context construction in human communication. PsyArXiv. https://doi.org/10.31234/osf.io/rwfe6

Stolk, A., Noordzij, M. L., Volman, I., Verhagen, L., Overeem, S., van Elswijk, G., Bloem, B., Hagoort, P., \& Toni, I. (2014). Understanding communicative actions: A repetitive TMS study. Cortex, 51, 25-34. https://doi.org/10.1016/j.cortex.2013.10.005

Stolk, A., Verhagen, L., \& Toni, I. (2016). Conceptual Alignment: How Brains Achieve Mutual Understanding. Trends in Cognitive Sciences, 20(3), 180-191. https://doi.org/10.1016/j.tics.2015.11.007

Tamariz, M. (2017). Experimental Studies on the Cultural Evolution of Language. Annual Review of Linguistics, 3(1), 389-407. https://doi.org/10.1146/annurev-linguistics-011516033807 
Tamariz, M., \& Kirby, S. (2016). The cultural evolution of language. Current Opinion in Psychology, 8, 37-43. https://doi.org/10.1016/j.copsyc.2015.09.003

Theisen, C. A., Oberlander, J., \& Kirby, S. (2010). Systematicity and arbitrariness in novel communication systems. Interaction Studies, 11(1), 14-32. https://doi.org/10.1075/is.11.1.08the

Thorpe, W. H. (1958). The Learning of Song Patterns by Birds, with Especial Reference to the Song of the Chaffinch Fringilla Coelebs. Ibis, 100(4), 535-570. https://doi.org/10.1111/j.1474-919X.1958.tb07960.x

Tinits, P., Nölle, J., \& Hartmann, S. (2017). Usage context influences the evolution of overspecification in iterated learning. Journal of Language Evolution, 2(2), 148-159. https://doi.org/10.1093/jole/lzx011

Tomasello, M. (2008). Origins of human communication. MIT Press.

Tomasello, M. (2014). A natural history of human thinking. Harvard University Press.

Tylén, K., Philipsen, J. S., Roepstorff, A., \& Fusaroli, R. (2016). Trails of meaning construction: Symbolic artifacts engage the social brain. NeuroImage, 134, 105-112. https://doi.org/10.1016/j.neuroimage.2016.03.056

Verhoef, T. (2012). The origins of duality of patterning in artificial whistled languages. Language and Cognition, 4(4), 357-380. https://doi.org/10.1515/langcog-2012-0019

Verhoef, T., Kirby, S., \& De Boer, B. (2014). Emergence of combinatorial structure and economy through iterated learning with continuous acoustic signals. Journal of Phonetics, 43, $57-68$.

Vihman, V.-A., Nelson, D., \& Kirby, S. (2018). Animacy Distinctions Arise from Iterated Learning. Open Linguistics, 4(1), 552-565. https://doi.org/10.1515/opli-2018-0027

Winters, J., Kirby, S., \& Smith, K. (2015). Languages adapt to their contextual niche. Language and Cognition, 7(3), 415-449. https://doi.org/10.1017/langcog.2014.35

Winters, J., \& Morin, O. (2019). From Context to Code: Information Transfer Constrains the Emergence of Graphic Codes. Cognitive Science, 43(3), e12722. https://doi.org/10.1111/cogs. 12722

Wolfle, D. L. (1933). The Relative Stability of First and Second Syllables in an Artificial Language. Language, 9(4), 313-315. https://doi.org/10.2307/409418

Zlatev, J., Wacewicz, S., Żywiczyński, P., \& van de Weijer, J. (2017). Multimodal-first or pantomime-first?: Communicating events through pantomime with and without vocalization. Interaction Studies, 18(3), 465-488. https://doi.org/10.1075/is.18.3.08zla 